\title{
FAULT DETECTION AND DIAGNOSIS OF HIGH SPEED SWITCHING DEVICES IN POWER INVERTER
}

\author{
R. B. Dhumale ${ }^{1}$, S. D. Lokhande ${ }^{2}$, N. D. Thombare ${ }^{3}$, M. P. Ghatule ${ }^{4}$ \\ ${ }^{l}$ Department of Electronics and Telecommunication Engineering, Sinhgad College of Engineering, Pune, India- \\ 411046 \\ ${ }^{2}$ Department of Electronics and Telecommunication Engineering, Sinhgad College of Engineering, Pune, India- \\ 411046 \\ ${ }^{3}$ Department of Electronics and Telecommunication Engineering, Sinhgad College of Engineering, Pune, India- \\ 411046 \\ ${ }^{4}$ Sinhgad College of Science, Pune, India-411046
}

\begin{abstract}
Power electronic based inverters are the major components in industry. A fault diagnostics framework composed of a pattern recognition system, having machine learning technology as its integral part is utilized for failure detection of different switches and tracing multiple types of faults in an inverter. Hardware point of view power electronics inverter can be considered to be the weakest link. Hence, this work is carried on detecting faults and classifies which switches in the inverter cause the fault. Diagnosis can help to avoid unplanned breakdown, to make possible to run an emergency operation in case of a fault. On the basis of theoretical foundations of electronic power inverter a simulation model has been developed to simulate the healthy condition and all single-switch open circuit faults. The generated signal is processed using Discrete Wavelet Transform (DWT) and Fuzzy Inference Logic (FIL). A smart and accurate classification of faults is obtained using simulation results, which are tested on a wide operation domain and various load conditions.
\end{abstract}

Keywords: Fault Diagnosis, DWT, Fuzzy Logic, Artificial Intelligence (AI).

\section{INTRODUCTION}

Automation process in the industry is electromechanical system, where the electrical drive is important and in parts is the central component. The costs of planned standstill of these automation systems can be very high and for unplanned standstill often much higher than for planned standstill. Breakdown or standstill of such many drives in the industry often leads to the complete malfunction of the system. Thus, continuous monitoring of this automation systems will led an economic operation a high utilization over time and short standstill times are required. Monitoring and diagnosis as well as preventive maintenance are valuable means to achieve this. All this depends on the economical conditions for the given system and its application. A large number of industrial drives are used in many applications including plants to rotate the conveyor belts, hybrid electric vehicles (HEV) and electric vehicles (EV). The electric drive systems more susceptible to high end risks. Due to fault occurrence at the input rectifier, or at the control sub-system, or at the power inverter stage. In general, when one of these faults occurs, the drive system has to be stopped for an un-programmed maintenance schedule to avoid catastrophic breakdown. An automated detection and an intelligent diagnosis of open circuit, short circuit and misfiring faults in solid state switches of power inverter system is therefore of crucial significance. Precise information and detailed knowledge about the behavior of faults makes huge contribution in constructing reliable and comprehensive systems. Introduction of such strategic subsystem as an integral part of motor drive systems requires practically conclusive performance of hardware and software. It must perform the tasks of fault detection, fault diagnosis and must apply corrective measures. An inverter can generate various types of faults that can be classified [9] as follows [1]:

- $\quad$ DC link short-circuit to ground (F1)

- $\quad$ Open-circuit damage of IGBT (F2)

- $\quad$ Short-circuit damage of IGBT (F3)

- $\quad$ DC link capacitor (F4)

- $\quad$ Single line to ground fault at machine terminal (F5)

The system discussed in this paper is based on combination of Discrete Wavelet Transformations (DWT) with Fuzzy Logic.

\section{VSI FAULT DIAGNOSIS METHODS}

Several methods have been developed to solve the fault diagnosis problem. The Wavelet-Fuzzy based algorithm shows the ability of AI for monitoring and diagnosis of power electronics system [2]. This system is effective if the fuzzy rules are carefully designed and it requires high implementation efforts [2]. The combination of Wavelet and Neural Network method reported less than 5\% error [3]. This method is implemented by using Back propagation Neural Network (NN) with three input, six hidden and one output neurons. Normalized DC values are fed to the $\mathrm{NN}$ for 
classification [3]. The combination of Continues Wavelet Transform (CWT) and Adaptive Neuro Fuzzy Interface System (ANFIS) requires high implementation efforts for training of NN [4].This system is implemented by using ' $\mathrm{db} 2$ ' mother wavelet and Zero Order Sugeno-type ANFIS [4]. Clustering-ANFIS (C-ANFIS) method is implemented to reduce the number of fuzzy rules and learning time [5]. CANFIS has reduced the dimension of fuzzy model and training time compared to other methods and worst case error $2.26 \%$ is reported [5].

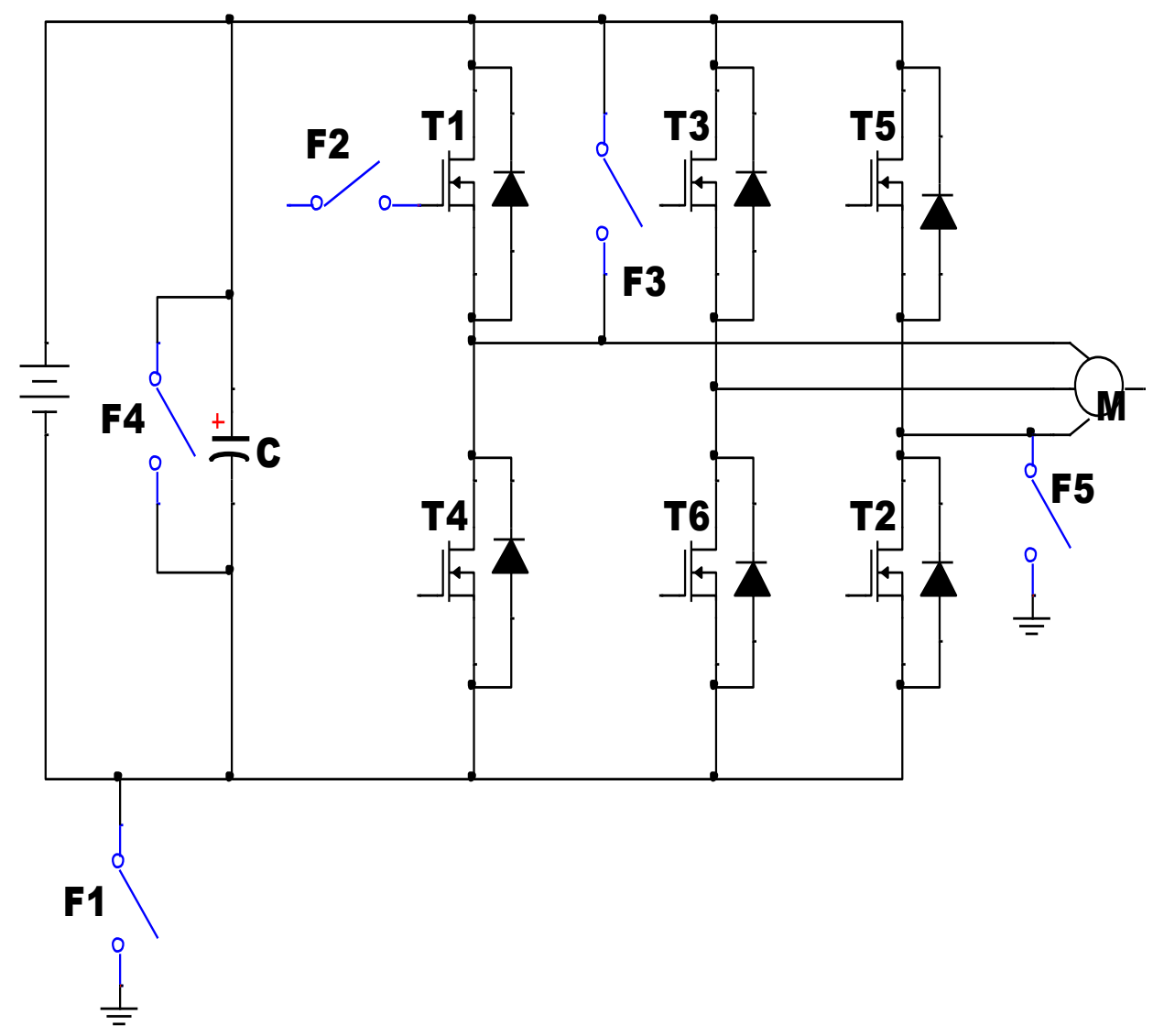

Fig -1: Three phase inverter faulty model

\section{DISCRETE WAVELET TRANSFORM}

\section{ANALYSIS}

In the signal processing, the DWT is a method to decompose an input signal of interest into a set of elementary waveforms, called wavelets and provides a way to analyze the signal by examining the coefficients of these wavelets. The Ability to perform local analysis is one of the major advantage of wavelets . Wavelet analyzes a localized area of a larger signal. Dislike other signal processing techniques wavelet analysis shoes higher competence in the area of analyzing patterns, breakdown points judgments and discontinuity examination. DWT helps in analysis and synthesis of original signal by reducing the computation time. Signal is analyzed by using filters at different frequencies and at different scales. Low Pass Filter (LPF) and High Pass Filter (HPF) are used in the analysis of low frequency signal and high frequency signal respectively. Resolution and scale of the signal is changed with the help of filtering and sampling operation respectively. Resolution measures the amount of detailed information in signal. Sampling may be Upsampling or Downsampling.
Upsampling is process of increasing sampling rate of signal. Downsampling operation is called as subsampling and it reduces the sampling rate of the signal [6].

\section{METHODOLOGY}

A combined DWT algorithm and Fuzzy Inference System (FIS) have been implemented to perform fault diagnosis of three phases VSI. The Figure-2 illustrates the block diagram of Fuzzy inference system. The implementation of DWTFIS based classifier requires three consecutive steps: Current Sensing, DWT and Classifier. DWT is used to detect the change in output parameter. The wavelet transform is capable of analyzing proportionate frequency changes with time and accordingly clever enough to sense and pinpoint short duration malfunctions. The fault occurred in system is determined by using Fuzzy Logic (FL) System. The structure of FIS classifier is defined based on the obtained rules, which are fuzzyfied in order to avoid the classification surface discontinuity. 


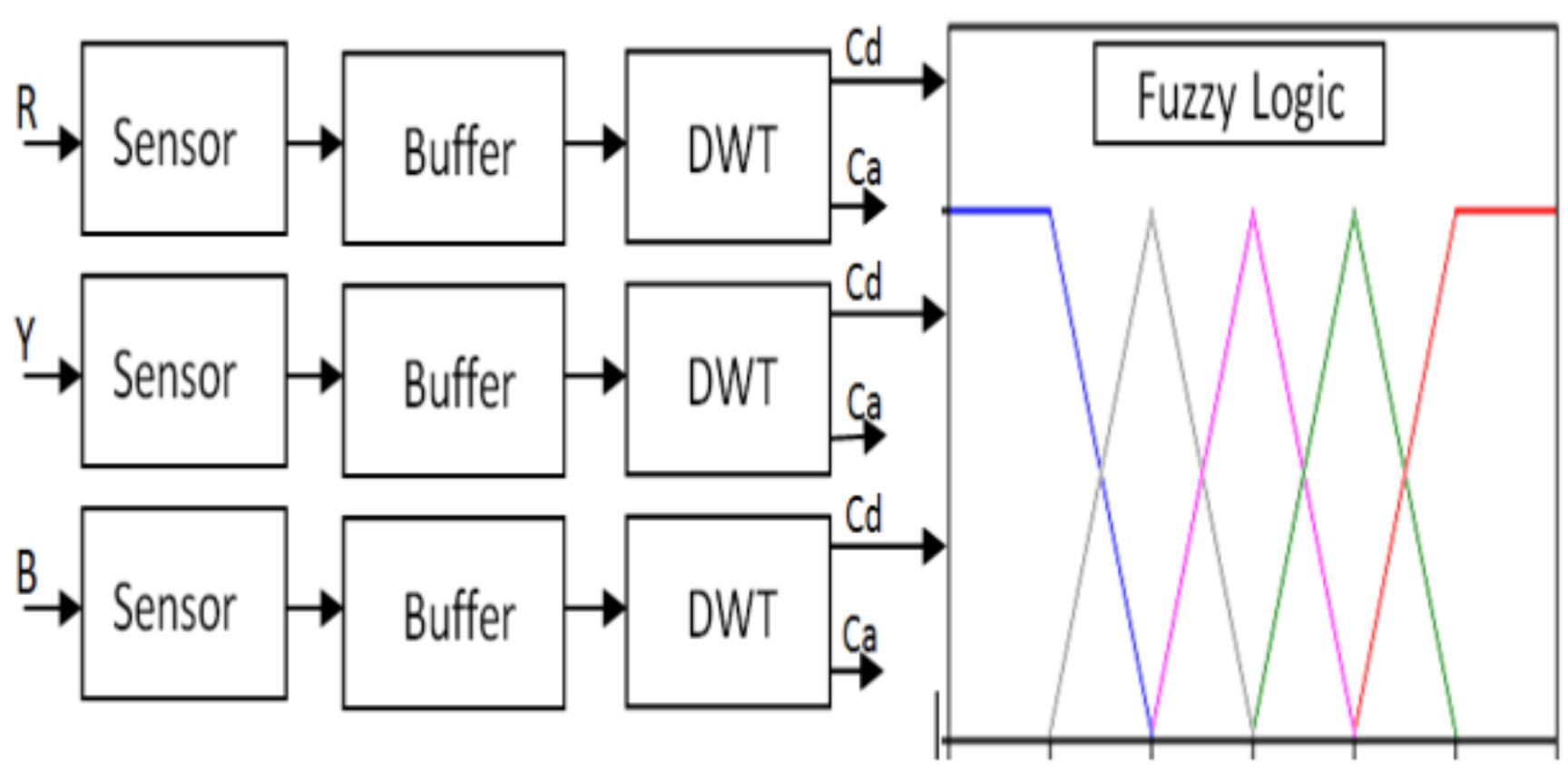

Fig -2: Block diagram of proposed system

Table -1: Rules for Fuzzy Logic

\begin{tabular}{|l|l|l|l|l|l|l|l|l|l|l|}
\hline \multirow{2}{*}{ Rule } & \multicolumn{9}{l}{ If } & \multicolumn{2}{l|}{ Then } \\
\cline { 2 - 14 } & Ia & Ib & Ic & G & T1 & T2 & T3 & T4 & T5 & T6 \\
\hline 1 & 0 & 0 & 0 & H & & & & & & \\
\hline 2 & $-\mathrm{Ve}$ & $+\mathrm{Ve}$ & $+\mathrm{Ve}$ & & $\mathrm{F}$ & & & & & \\
\hline 3 & $-\mathrm{Ve}$ & $-\mathrm{Ve}$ & $+\mathrm{Ve}$ & & & $\mathrm{F}$ & & & & \\
\hline 4 & $+\mathrm{Ve}$ & $-\mathrm{Ve}$ & $+\mathrm{Ve}$ & & & & $\mathrm{F}$ & & & \\
\hline 5 & $+\mathrm{Ve}$ & $-\mathrm{Ve}$ & $-\mathrm{Ve}$ & & & & & $\mathrm{F}$ & & \\
\hline 6 & $+\mathrm{Ve}$ & $+\mathrm{Ve}$ & $-\mathrm{Ve}$ & & & & & & $\mathrm{F}$ & \\
\hline 7 & $-\mathrm{Ve}$ & $+\mathrm{Ve}$ & $-\mathrm{Ve}$ & & & & & & & $\mathrm{F}$ \\
\hline
\end{tabular}
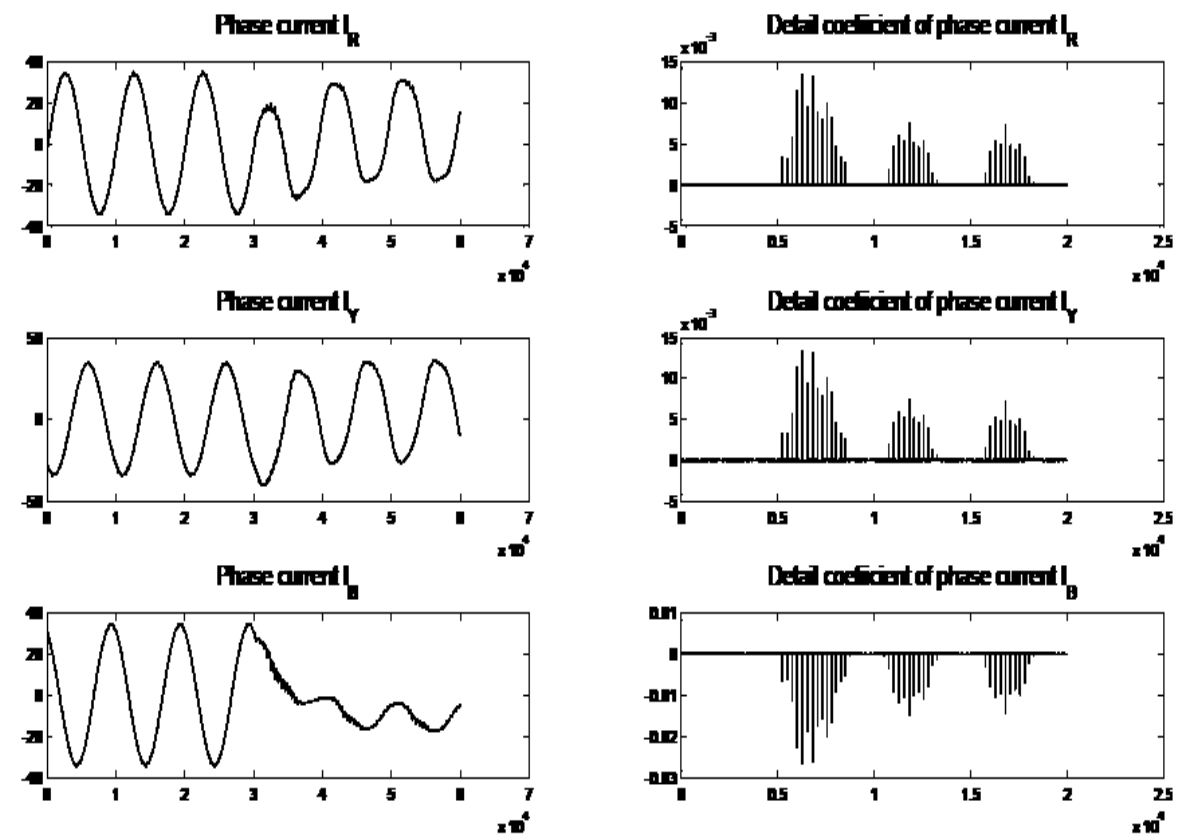

Fig -3: Transistor T5 open circuit fault 


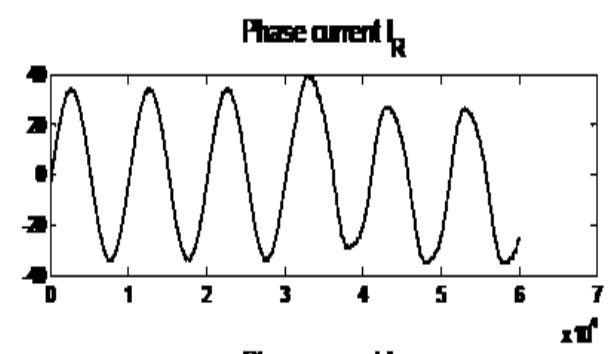

Phasednit/

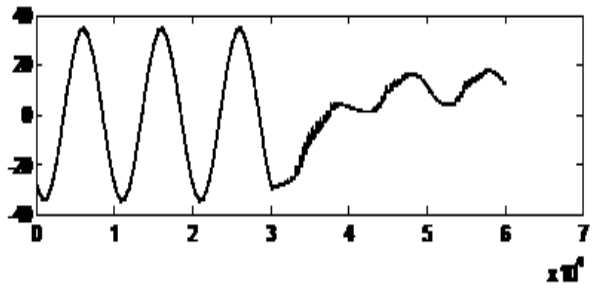

Phasednith

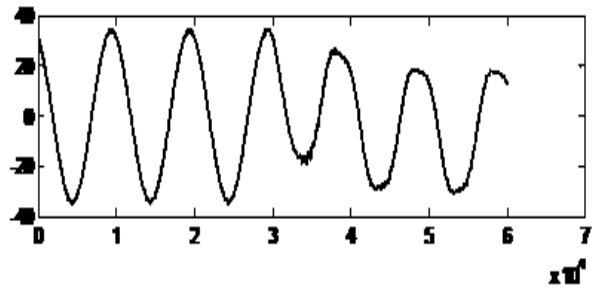

Det: coeleivil of phase ancith

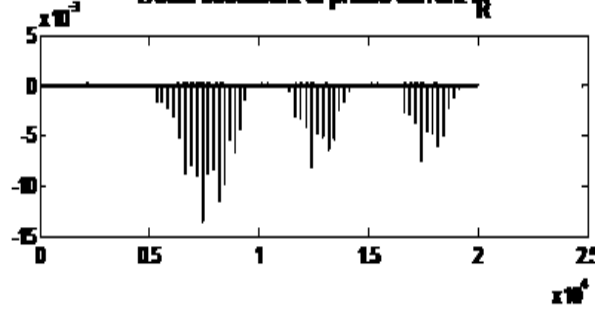

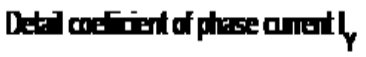

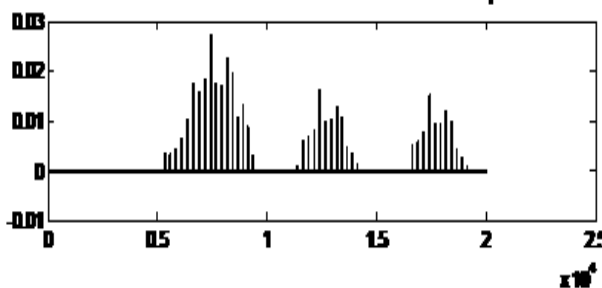

Det weicit of phesenmith

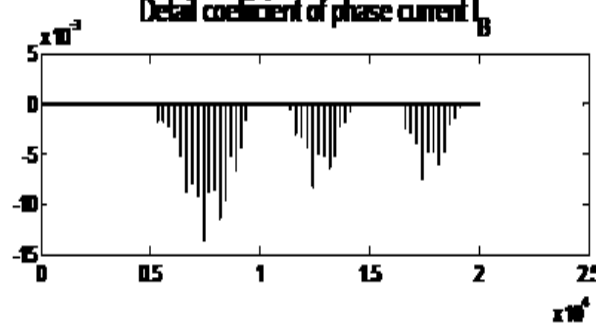

Fig -4: Transistor T6 open circuit fault

Faults in R Phase

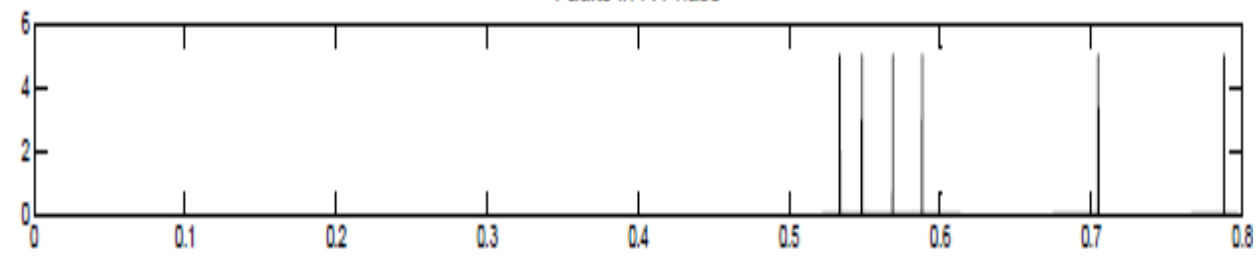

Faults in Y Phase

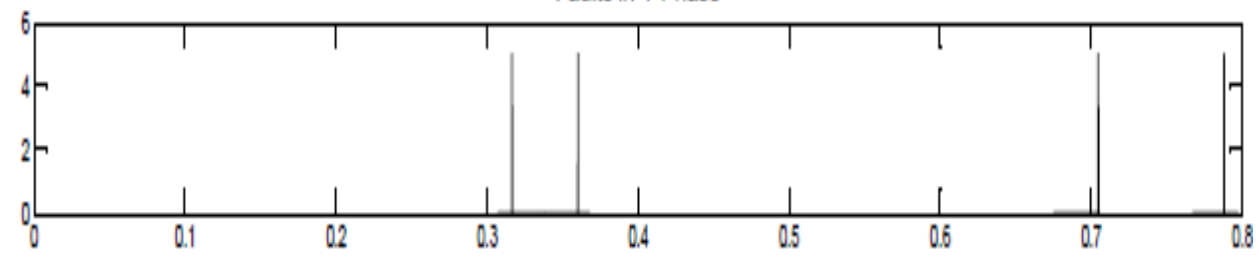

Faults in B Phase

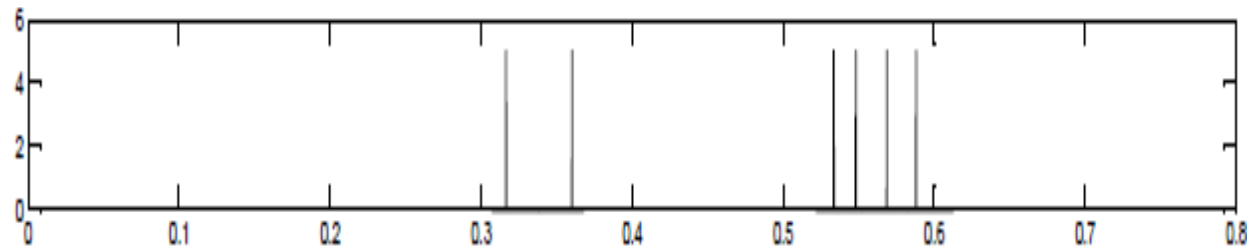

Output of FUZZY

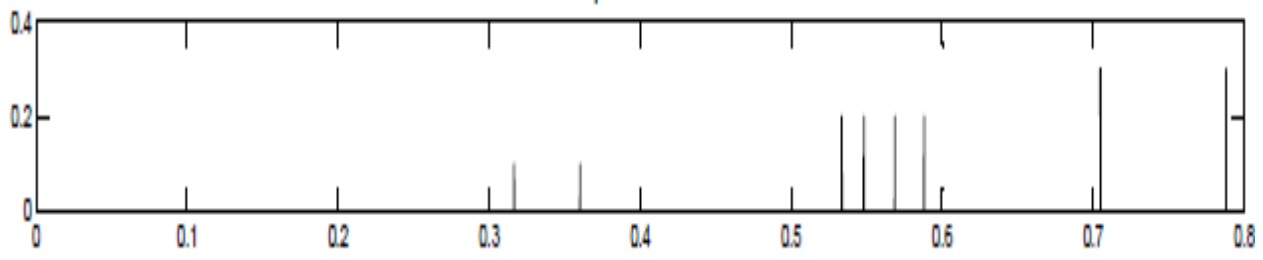

Fig- 5: Classification of faults using Fuzzy Logic 


\section{SIMULATION RESULTS}

The proposed system will find which transistor is faulty. The Figure-3 and Figure-4 illustrate, if T5 is faulty then DC coefficient of current $\mathrm{Ia}, \mathrm{Ib}$ and Ic waveform are positive, positive and negative respectively. For faulty transistor T6 DC coefficients of current waveform Ia, Ib and Ic are negative, negative and positive respectively. Depending on such observation some rules are implemented for Fuzzy Logic as shown in Table-1. By observing the input and output data set Fuzzy rules and membership functions are constructed. The membership functions will be generated for zero, phase current with positive DC offset $(+\mathrm{Ve})$ and phase current with negative DC offset negative (-Ve). In table ' $\mathrm{H}$ ' and ' $\mathrm{F}$ ' indicates healthy system and faulty transistor respectively. The signal always contains noise and disturbance. Phase currents may bring in a low level DC offset with a slight load unbalance. In such cases fuzzy rules should be carefully designed. The Fuzzy Logic System is implemented in MATLAB.

Firstly, the fault introduced in single switch T5 or T6 is an open-circuit fault. Load current waveform and detail coefficient for single switch T5 and T6 fault are shown in Figure 2 and 3 respectively. It must be stated that phase currents has the dc offset initiated due to this fault. The amount of dc offset is sensed in this faulty phase is high in contrast to the other faulty phases. Also, detailed coefficient's polarities (Positive, Negative or Zero) in this faulty phase and the other two phases are distinguished. Discontinuity and sharp change in current waveforms are easily visible in DWT coefficient sharing same specific pattern. The feature of localization of the exact instant of the fault occurrence is very important. Feature extraction of DC coefficients from the three phase currents shown in Figure3. Output of current sensors is given to Buffer of size 64 to convert continuous current waveform into discrete form. The output of Buffer is connected to DWT block to calculate Detailed Coefficients (DC) value. DB2 filter at level 4 is used to calculate DC value. The threshold of 0.005 is set by observing various samples. If the DC value is greater than 0.005 then open circuit fault or misfiring Fault is occurred, hence the output DC value from DWT is compared with 0.005 and if it is greater than 0.005 the output of comparator will be 1 . For further operation the value of comparator is multiplied by 5 by using embedded MATLAB function. The Fuzzy logic is used for fault classification. Fuzzy logic is designed such that input from system is less than 5 then membership function provides zero value and if greater than or equals to 5 then membership function provides one value. The classification of faults using fuzzy logic is shown in Figure-5. The output membership function of Fuzzy logic is designed such that if $\mathrm{T} 1$ is faulty, then output will be 0.1 . If $\mathrm{T} 2$ is faulty, then output will be 0.2 and if T3 is faulty then output will be 0.3 .

\section{CONCLUSION}

The implemented approach is found to be efficient in accurate identification and isolation of faults. The approach is capable of identifying the fault without any ambiguity. A model of the electric drive inverter with a three-phase VSI and a control mechanism is developed that successfully simulates the normal operations of the power electronics inverter, six single switch open fault conditions. This model has been implemented using a simulation toolbox of MATLAB to generate signals for Fuzzy Logic based diagnosis of VSI. The simulated results shows that wavelet transform together fuzzy logic will capable of monitoring and classification of open circuit fault in power electronic drives.

\section{REFERENCES}

[1]. R. L. A. Ribeiro, C. B. Jacobina, E. R. C. Silva, and A. M. N. Lima, "Fault detection of open-switch damage in voltage-fed PWM motor drive systems," IEEE Trans. Power Electron., vol. 18, no. 2, pp. 587-593, Mar. 2003.

[2]. M. R. Mamat, M. Rizon, and M. S. Khanniche, "Fault detection of 3-phase VSI using wavelet-fuzzy algorithm," Amer. J. Appl. Sci., vol. 3,no. 1, pp. 1642-1648, 2006.

[3]. F. Charfi, F. Sellami, and K. Al-Haddad, "Fault diagnosis in power system using wavelet transforms and neural networks," in Proc. IEEE Int. Symp. Ind. Electron, 2006, pp. 1143-1148.

[4]. M. A. Awadallah and M. M. Morcos, "Diagnosis of switch open circuit fault in PM brushless dc motor drives," in Proc. Large Eng. Syst. Conf. Power Eng., May 2003, pp. 69-73.

[5]. J. H. Park, D. H. Kim, S. S. Kim, D. J. Lee, and M. G. Chun, "C-ANFIS based fault diagnosis for voltage-fed PWM motor drive systems," in Proc. Annu. Meeting North Amer. Fuzzy Inform. Soc., 2004,pp. 379-383.

[6]. Roby Polikar, "The Wavelet Tutorial, Part IV, and Multiresolution Analysis: The Discrete wavelet transform". 